\title{
Hypoxia-inducible factor-1 $\alpha$ regulates epithelial-to-mesenchymal transition in paraquat-induced pulmonary fibrosis by activating lysyl oxidase
}

\author{
JIAN LU ${ }^{1}$, YONGBING QIAN ${ }^{1}$, WEI JIN ${ }^{1}$, RUI TIAN ${ }^{1}$, YONG ZHU $^{2}$, JINFENG WANG $^{2}$, \\ XIAOXIAO $\mathrm{MENG}^{2}$ and RUILAN WANG ${ }^{1}$ \\ ${ }^{1}$ Department of Critical Care Medicine, Shanghai General Hospital of Nanjing Medical University; \\ ${ }^{2}$ Department of Critical Care Medicine, Shanghai General Hospital, Shanghai Jiaotong University, \\ School of Medicine, Shanghai 201620, P.R. China
}

Received May 12, 2017; Accepted October 25, 2017

DOI: $10.3892 /$ etm.2017.5677

\begin{abstract}
Pulmonary fibrosis (PF) is one of the most prevalent causes of death following paraquat (PQ) poisoning. As demonstrated in previous studies by the present authors, epithelial-to-mesenchymal transition (EMT) is associated with PQ-induced PF. In addition, hypoxia-inducible factor-1 $\alpha$ $(\mathrm{HIF}-1 \alpha)$ and lysyl oxidase (LOX) promote EMT following PQ poisoning. However, the association between HIF-1 $\alpha$ - and LOX-mediated regulation of EMT remains unclear. The present study investigated the association between HIF-1 $\alpha$ and LOX with regard to PQ-induced EMT. A549 and RLE-6TN cells were treated with PQ, and HIF-1 $\alpha$ and LOX expression was silenced with short interfering RNAs. Changes in the expression of HIF-1 $\alpha$, LOX, $\beta$-catenin and EMT-related makers were detected using real-time quantitative polymerase chain reaction, immunofluorescence, and western blotting. HIF-1 $\alpha$ and LOX were associated with PQ-induced EMT, and their expression levels were significantly increased $(\mathrm{P}<0.05)$. LOX expression was significantly decreased following PQ poisoning when HIF-1 $\alpha$ expression was inhibited $(\mathrm{P}<0.05)$. However, the level of HIF-1 $\alpha$ did not change significantly when LOX was silenced. The expression level of $\beta$-catenin and the degree of EMT were significantly decreased following HIF-1 $\alpha$ and LOX silencing in both cell lines $(\mathrm{P}<0.05)$. The association between HIF-1 $\alpha$ and LOX in regulating EMT during PQ-induced PF may be unidirectional. HIF-1 $\alpha$ may regulate $\mathrm{PQ}$-induced EMT through the LOX/ $/$-catenin pathway.
\end{abstract}

Correspondence to: Dr Ruilan Wang, Department of Critical Care Medicine, Shanghai General Hospital of Nanjing Medical University, 650 Xin Songjiang Road, Shanghai 201620, P.R. China E-mail:wangyusun@hotmail.com

Abbreviations: EMT, epithelial-to-mesenchymal transition; HIF- $1 \alpha$, hypoxia-inducible factor- $1 \alpha$; LOX, lysyl oxidase; PF, pulmonary fibrosis; PQ, paraquat; $\alpha$-SMA, $\alpha$-smooth muscle actin

Key words: hypoxia-inducible factor- $1 \alpha$, lysyl oxidase, epithelial-tomesenchymal transition, paraquat, pulmonary fibrosis

\section{Introduction}

Paraquat (PQ) has been one of the most effective and widely used herbicides over the last few decades, particularly in rural areas of developing countries; however, PQ poisoning has become a serious problem, with reports of mortality $>90 \%(1,2)$. The primary pathological effects of PQ are observed in the lung, where pulmonary concentrations are 6-10 times higher than in plasma following PQ ingestion (3). Furthermore, PQ accumulates in the lungs as blood levels begin to decrease (3). The rapid accumulation of PQ damages the parenchymal cells in the lung and induces the excessive repair of lung tissues, which results in irreversible and extensive pulmonary fibrosis (PF) (3) and eventually leads to high mortality rates. However, the exact mechanism that leads to toxicity remains unclear, and no specific therapy has been recommended.

Epithelial-to-mesenchymal transition (EMT) occurs in multiple contexts, including embryonic development, tissue fibrosis, and cancer. EMT is defined as the process by which stationary epithelial cells (identified by high levels of E-cadherin and zonula occludens-1, which are markers of epithelial cells) undergo phenotypic changes, including the loss of cell-cell adhesion and apical-basal polarity, and acquire mesenchymal characteristics, including high levels of $\alpha$-smooth muscle actin ( $\alpha$-SMA) and $\mathrm{N}$-cadherin (markers of mesenchymal cells), that confer migratory capacity $(4,5)$. According to previous findings, EMT has an important role in the development of PF. Alveolar epithelial cells could acquire mesenchyme cell phenotypes through EMT, these cells could then increase the deposition of extracellular matrix and further promote the development of PF (5-7). Furthermore, EMT has been demonstrated to serve an important role in $\mathrm{PQ}$-induced $\mathrm{PF}$ in recent studies by the present authors $(8,9)$.

Hypoxia-inducible factor- $1 \alpha(\mathrm{HIF}-1 \alpha)$ has roles in tumorigenesis, inflammation, and cell metabolism in hypoxia, and its expression is correlated with a variety of fibrotic diseases $(10,11)$. HIF-1 $\alpha$ has also been demonstrated to induce EMT and contribute to $\mathrm{PF}(12,13)$. Previous studies have detected an early increase in HIF-1 $\alpha$ expression following PQ poisoning and revealed that HIF-1 $\alpha$ modulates EMT in cases of PF $(9,14)$. 
Lysyl oxidase (LOX) is a secreted copper-dependent amine oxidase that is important for growth, stabilization, remodeling and repair. Its primary function is to catalyze the covalent cross-linking of collagens and elastin in the extracellular matrix, although it also has intracellular functions (15). LOX participates in various fibrosis processes, such as lung, myocardial and renal fibrosis (16-18). As demonstrated in a previous study by the present authors, LOX promotes EMT in PQ-induced PF (8). LOX was previously considered a critical target of HIF-1 $\alpha$ (19); however, HIF-1 $\alpha$ and LOX have since been demonstrated to provide bidirectional regulation of colon and ovarian carcinomas $(20,21)$. The potential for dual regulation via HIF-1 $\alpha$ and LOX remains controversial, particularly in PQ-induced PF. The present study investigated the association between HIF-1 $\alpha$ and LOX with regard to PQ-induced PF.

\section{Materials and methods}

Reagents. PQ powder was obtained from Sigma-Aldrich (Merck KGaA, Darmstadt, Germany). Anti-HIF-1 $\alpha$ antibodies (cat. no. BS3514) were purchased from Bioworld Technology, Inc. (St. Louis Park, MN, USA). Anti-LOX (cat. no. ab174316), anti-E-cadherin (cat. no. ab184633) and anti- $\alpha-S M A$ (cat. no. ab7817) primary antibodies were obtained from Abcam (Cambridge, MA, USA). Anti- $\beta$-catenin (cat. no. 8480) and anti-GAPDH (cat. no. 5174) antibodies were purchased from Cell Signaling Technology, Inc. (Boston, MA, USA). Horseradish peroxidase-conjugated anti-rabbit immunoglobulin (Ig)G (cat. no. A0208), anti-mouse IgG secondary antibodies (cat. no. A0216), immunofluorescence staining kits with Alexa Fluor 647-labeled goat anti-rabbit immunoglobulin G (cat. no. A0468) and kits with Alexa Fluor 488-labeled goat anti-rabbit IgG (cat. no. A0423) were obtained from Beyotime Institute of Biotechnology (Shanghai, China).

Cell culture. Human lung adenocarcinoma epithelial cells (A549) and rat alveolar type II cells (RLE-6TN) were obtained from the American Type Culture Collection (Manassas, VA, USA). A549 cells were cultured in Dulbecco's modified Eagle's medium (DMEM) (HyClone; GE Healthcare Life Sciences, Logan, UT, USA) supplemented with $10 \%$ fetal bovine serum (Gibco; Thermo Fisher Scientific, Inc., Waltham, MA, USA) and a $1 \%$ antibiotic solution $(100 \mathrm{U} / \mathrm{ml}$ penicillin and $0.1 \mathrm{mg} / \mathrm{ml}$ streptomycin). RLE-6TN cells were cultured in DMEM/nutrient mixture F-12 supplemented with $10 \%$ FBS and $1 \%$ antibiotic solution. Both cell lines were cultured at $37^{\circ} \mathrm{C}$ in an atmosphere containing $5 \% \mathrm{CO}_{2}$. Cells were subsequently treated with PQ (at a concentration of $800 \mu \mathrm{mol} / 1$ for A549 cells and $160 \mu \mathrm{mol} / 1$ for RLE-6TN cells) for $24 \mathrm{~h}$ at $37^{\circ} \mathrm{C}$. These concentrations were used in accordance with a recent study by the present authors (9). The effect of HIF-1 $\alpha$ or LOX silencing on cells was detected and the expression of other proteins was subsequently assessed using western blotting.

Reverse transcription-quantitative polymerase chain reaction (RT-qPCR). Total RNA was isolated from cells using TRIzol (Invitrogen; Thermo Fisher Scientific, Inc.). The total RNA concentration was determined using an ultraviolet spectrophotometer. Reverse transcription was performed using a PrimeScript RT Master Mix kit (Takara Biotechnology Co.,
Ltd., Dalian, China), according to the manufacturer's instructions. Real-time quantitative PCR was performed using a SYBR Premix Ex Taq kit (Takara Biotechnology Co., Ltd.) in a ViiA 7 PCR system. Sangon Biotech Co., Ltd. (Shanghai, China) generated the primers for HIF- $1 \alpha$, LOX, $\beta$-catenin and $\beta$-actin. Primer sequences are listed in Table I. The thermocycling conditions were as follows: $2 \mathrm{~min}$ at $95^{\circ} \mathrm{C}$ for initial denaturation, followed by 40 amplification cycles consisting of $95^{\circ} \mathrm{C}$ for $10 \mathrm{sec}$ (denaturation), $60^{\circ} \mathrm{C}$ for $30 \mathrm{sec}$ (anneal) and $72^{\circ} \mathrm{C}$ for $30 \mathrm{sec}$ (extension). The method of quantification used was the $2^{-\Delta \Delta \mathrm{Cq}}$ method (22). Each assay was performed in triplicate, and $\beta$-actin served as a loading control.

Western blotting. Total proteins were harvested from both cell lines in each group and lysed using radioimmunoprecipitation assay buffer (Beyotime Institute of Biotechnology). Protein concentrations were determined using a bicinchoninic acid protein assay kit (Beyotime Institute of Biotechnology). Total protein samples $(\sim 30 \mu \mathrm{g}$ per lane) were separated via $8 \%$ SDS-PAGE (Beyotime Institute of Biotechnology), transferred to polyvinylidene difluoride membranes (Bio-Rad Laboratories, Inc., Hercules, CA, USA), blocked with 5\% skimmed milk in Tris-buffered saline containing Tween-20 (TBST) for $90 \mathrm{~min}$ at room temperature (RT), and incubated with antibodies against HIF-1 $\alpha$ (1:500), LOX (1:1,000), E-cadherin (1:500), $\alpha$-SMA $(1: 500), \beta$-catenin $(1: 1,000)$ or GAPDH $(1: 500)$ overnight at $4^{\circ} \mathrm{C}$. Membranes were subsequently incubated with horseradish peroxidase-conjugated anti-rabbit $\mathrm{IgG}$ or anti-mouse IgG secondary antibodies (1:2,000; Beyotime Institute of Biotechnology) at RT. Following three washes with TBST, proteins were observed using a highly sensitive enhanced chemiluminescent agent (Thermo Fisher Scientific, Inc.). The band intensity was determined using ImageJ software (version 10.2; National Institutes of Health, Bethesda, MD, USA).

Immunofluorescence staining. Both cell lines were cultured in confocal dishes for $24 \mathrm{~h}$ at $37^{\circ} \mathrm{C}$ and incubated with PQ for $24 \mathrm{~h}$ at $37^{\circ} \mathrm{C}$. Cells were washed with PBS, fixed with $4 \%$ paraformaldehyde (Sigma-Aldrich; Merck KGaA) for $10 \mathrm{~min}$ at RT, permeabilized with $0.5 \%$ Triton X100 (Sigma-Aldrich; Merck $\mathrm{KgaA}$ ) for $10 \mathrm{~min}$ and blocked with $5 \%$ bovine serum albumin (1 $\mathrm{g}$ bovine serum albumin powder and $20 \mathrm{ml}$ Tris-buffered saline; Beyotime Institute of Biotechnology) for $1 \mathrm{~h}$ at RT. Subsequently, cells were incubated with anti-LOX $(1: 100)$ or anti-HIF-1 $\alpha$ (1:50) primary antibodies overnight at $4^{\circ} \mathrm{C}$. Following three washes with TBST, cells were incubated with immunofluorescence staining kits with Alexa Fluor 647-labeled goat anti-rabbit IgG (1:200) and kits with Alexa Fluor 488-labeled goat anti-rabbit IgG (1:200) for $1.5 \mathrm{~h}$ at RT. Nuclei were stained with DAPI (Beyotime Institute of Biotechnology) for $3 \mathrm{~min}$ at RT. Fluorescent signals were detected with a laser confocal scanning microscope (Leica TCS SP8; Leica Microsystems $\mathrm{GmbH}$, Wetzlar, Germany) and the cellular morphology was observed with a phase contrast microscope (AMEX1200, Thermo Fisher Scientific, Inc.) was used to observe the change of cellular morphology.

Transient transfection. A549 and RLE-6TN cells were cultured in 6-well culture plates as described above and divided into dimethyl sulfoxide groups (including the control, sicontrol, 
Table I. Primer sequences used in reverse transcription-quantitative polymerase chain reaction.

Gene

Species

Human

Human

Rat
Sequence (5'-3')

GTCTGAGGGGACAGGAGGAT
CTCCTCAGGTGGCTTGTCAG
CAACCTGAGATGCGCGG
GGTCGGCTGGGTAAGAAATC
CGTTTCGCCTTCATTATGGACTACCT
GCCGCTGGGTGTCCTGATGT
CTGGAACGGTGAAGGTGACA
AAGGGACTTCCTGTAACAATGCA
AAGTCTAGGGATGCAGCACG
AGATGGGAGCTCACGTTGTG
CCTACTACATCCAGGCATCCA
AGTCTCTGACATCCGCCCTA
GTGCAATTCCTGAGCTGACC
CGGGCTGTTTCTACGTCATT
CCTCTATGCCACACAGT
AGCCACCAATCCACACAG

HIF-1 $\alpha$, hypoxia-inducible factor-1 $\alpha$; LOX, lysyl oxidase; F, forward; R, reverse.

siHIF-1 $\alpha$ and siLOX groups) and PQ groups (including the control + PQ, sicontrol + PQ, siHIF-1 $\alpha+\mathrm{PQ}$ and siLOX + PQ groups).HIF-1 $\alpha$ and LOX short interfering (si)RNAs and negative control sequences were purchased from Shanghai GenePharma Co., Ltd. (Shanghai, China) and are listed in Table II. For transfection of each siRNA, $4 \mu$ l Lipofectamine ${ }^{\circledR} 2000$ (Invitrogen; Thermo Fisher Scientific, Inc.) were incubated with 100 pmol siRNA or negative control sequences in $500 \mu 1$ Opti-MEM medium (Gino Biomedical Technology Co., Ltd., Hangzhou, China) for $20 \mathrm{~min}$ at RT. Cells were transfected by replacing the medium with $2 \mathrm{ml}$ Opti-MEM medium containing the siRNA or negative control sequences and Lipofectamine ${ }^{\circledR} 2000$, and then incubating them at $37^{\circ} \mathrm{C}$ in a humidified atmosphere of $5 \% \mathrm{CO}_{2}$ for $6 \mathrm{~h}$. The Opti-MEM medium was then replaced with $2 \mathrm{ml}$ fresh culture medium. Subsequently, the cells in the PQ groups were incubated with PQ for $24 \mathrm{~h}$ and the other cells were treated with phosphate buffered saline. The total time from the start of transfection to subsequent experimentation was $48 \mathrm{~h}$.

Statistical analyses. Data were analyzed using SPSS (version 16.0; SPSS, Inc., Chicago, IL, USA) and expressed as the mean + standard deviation of triplicate experiments. Comparisons between two groups were performed using a Student's t-test and comparisons of multiple groups were performed using one-way analysis of variance and Dunnett's post hoc test. $\mathrm{P}<0.05$ was considered to indicate a statistically significant difference.

\section{Results}

HIF-1 $\alpha$ and LOX may regulate PQ-induced EMT. PQ treatment induced a significant decrease in E-cadherin expression and significantly increased $\alpha$-SMA expression as determined by western blotting (Fig. 1A), which confirmed
Table II. Sequences of siRNAs used for transfection.

\begin{tabular}{lcc}
\hline Species & siRNA & \multicolumn{1}{c}{ Sequence (5'-3') } \\
\hline Human & HIF-1 $\alpha$ & F: GCCGAGGAAGAACUAUGAATT \\
& & R: UUCAUAGUUCUUCCUCGGCTT \\
& LOX & F: CAGGCGAUUUGCAUGUACUTT \\
& & R: AGUACAUGCAAAUCGCCUGTT \\
Rat & HIF-1 $\alpha$ & F: GGGCCGUUCAAUUUAUGAATT \\
& & R: UUCAUAAAUUGAACGGCCCTT \\
& LOX & F: CCGGAUGUUAUGAUACUUATT \\
& & R: UAAGUAUCAUAACAUCCGGTT
\end{tabular}

Si, silencing; HIF, hypoxia-inducible factor; LOX, lysyl oxidase; F, forward; R, reverse.

that EMT participated in PQ-induced PF. Protein levels of HIF- $1 \alpha$ and LOX were significantly increased in the PQ groups compared with the control groups (Fig. 1B). Based on the immunofluorescence staining, the levels of HIF-1 $\alpha$ and LOX were markedly increased with $24 \mathrm{~h}$ of treatment with PQ compared with control groups (Fig. 1C). These results suggested that EMT served an important role in PQ-induced $\mathrm{PF}$, and that HIF-1 $\alpha$ and LOX may regulate EMT following $\mathrm{PQ}$ poisoning.

HIF-1a may promote EMT by upregulating LOX expression. The levels of HIF-1 $\alpha$, LOX- and EMT-related markers in PQ-poisoned A549 and RLE-6TN cells were measured following HIF-1 $\alpha$ silencing to determine the potential roles of HIF-1 $\alpha$ and LOX in PQ-induced EMT. HIF-1 $\alpha$ mRNA 
A

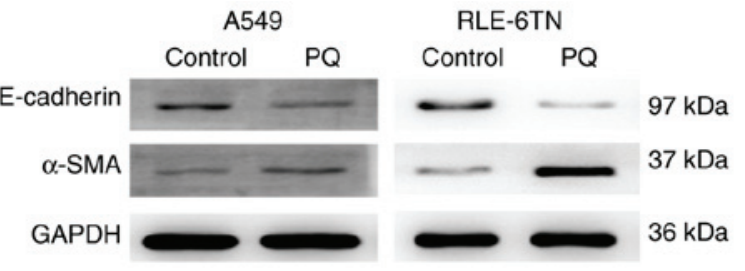

B

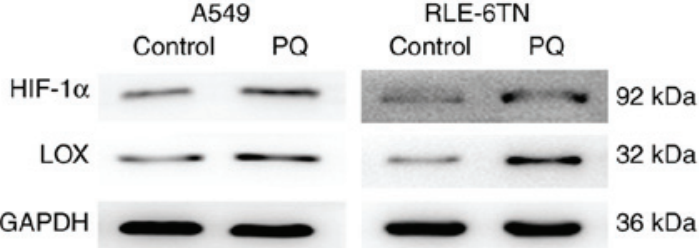

C

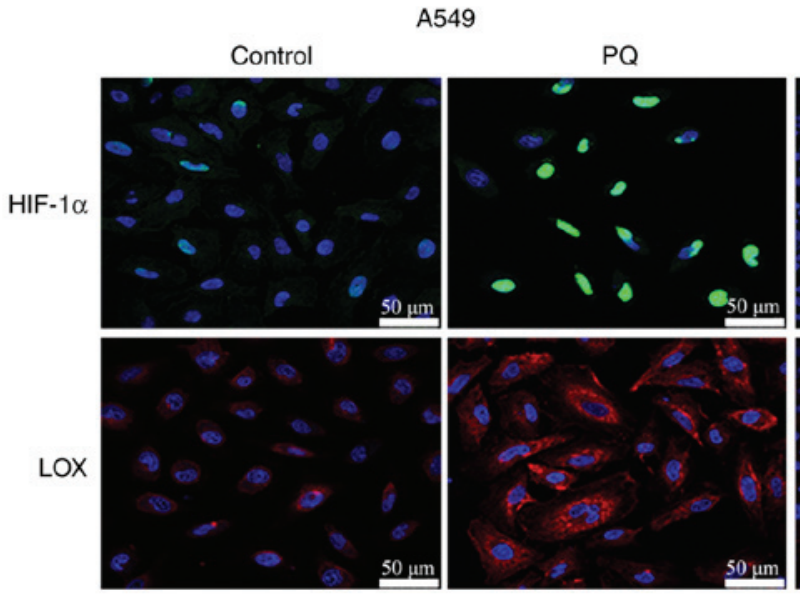

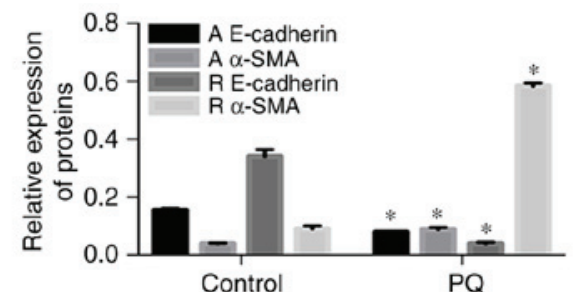

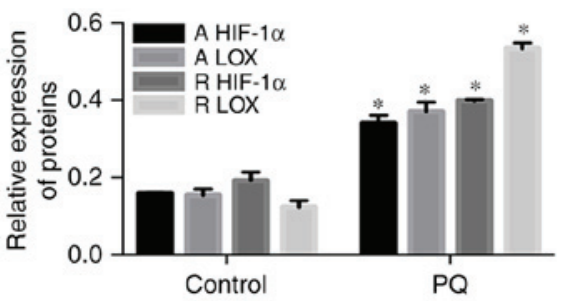

RLE-6TN

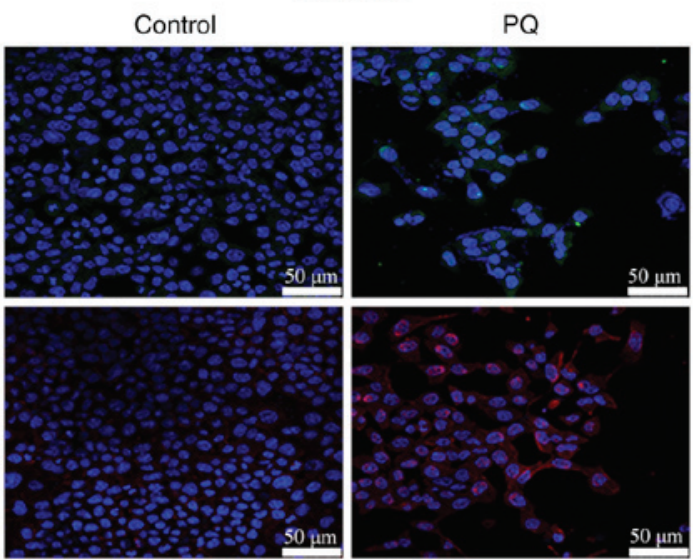

Figure 1. Levels of the epithelial-to-mesenchymal transition-associated proteins, HIF-1 $\alpha$ and LOX, increase during PQ-induced pulmonary fibrosis. (A) E-cadherin, $\alpha$-SMA and GAPDH levels were detected by western blotting. GAPDH was used as a loading control. (B) HIF-1 $\alpha$, LOX and GAPDH levels were detected by western blotting. (C) Levels of HIF-1 $\alpha$ and LOX proteins in A549 and RLE-6TN cells were detected by immunofluorescence staining. Scale bars, $50 \mu \mathrm{m}$. Data are presented as the mean + standard deviation $(\mathrm{n}=3)$. * $\mathrm{P}<0.05$ vs. control. HIF-1 $\alpha$, hypoxia-inducible factor-1 $\alpha$; LOX, lysyl oxidase; PQ, paraquat; $\alpha$-SMA, $\alpha$-smooth muscle actin; A, levels in A549 cells; R, levels in RLE-6TN cells.

expression was significantly decreased in the siHIF-1 $\alpha+\mathrm{PQ}$ group compared with the sicontrol + PQ group (Fig. 2A). The expression of EMT markers was reversed following HIF-1 $\alpha$ silencing, as $\alpha$-SMA expression decreased and E-cadherin expression increased (Fig. 2B). In addition, phase-contrast microscopy revealed that the morphology of cells in the PQ groups changed from a polygon to fusiform morphology compared with the control group. However, these changes were alleviated following HIF-1 $\alpha$ silencing (Fig. 2C). The level of LOX mRNA was significantly decreased in the siHIF-1 $\alpha+$ PQ group compared with the sicontrol + PQ group (Fig. 2D). The protein expression of LOX was reduced in the siHIF-1 $\alpha+P Q$ group compared with the sicontrol + PQ group (Fig. 2E). Therefore, HIF-1 $\alpha$ may have an important function in modulating PQ-induced EMT by inducing LOX expression.

LOX promotes $P Q$-induced EMT independently from HIF-1 $\alpha$. Levels of HIF-1 $\alpha$, LOX and EMT markers in PQ-poisoned cells following LOX silencing were subsequently determined. The level of LOX mRNA was significantly decreased in the siLOX group compared with the sicontrol group and in the siLOX + PQ group compared with the sicontrol + PQ group
(Fig. 3A). The expression of EMT markers was also reversed following LOX silencing, as $\alpha$-SMA expression decreased, and E-cadherin increased (Fig. 3B). In addition, phase-contrast microscopy revealed that the morphological changes (the degree of fusiformity was reduced) observed in cells in the PQ groups were alleviated following LOX silencing (Fig. 3C). However, the expression of HIF-1 $\alpha$ mRNA was not significantly changed in the siLOX + PQ group compared with the sicontrol + PQ group (Fig. 3D). Levels of HIF-1 $\alpha$ protein were also not significantly decreased following LOX expression inhibition (Fig. 3E). These findings suggest that LOX may promote PQ-induced EMT, but it does not regulate HIF-1 $\alpha$ expression.

HIF-1 a may regulate $P Q$-induced EMT through the $L O X / \beta$-catenin pathway. Changes in the levels of $\beta$-catenin were detected following HIF-1 $\alpha$ (LOX) inhibition in vitro to further reveal the interactions between HIF-1 $\alpha$, LOX and $\beta$-catenin. $\beta$-catenin mRNA levels in the PQ groups were significantly decreased following HIF-1 $\alpha$ (LOX) silencing (Fig. 4A and B). Similar results were observed for the protein expression of $\beta$-catenin (Fig. 4C and D). These findings suggest that HIF-1 $\alpha$ may modulate PQ-induced EMT via the $\mathrm{LOX} / \beta$-catenin pathway. 
A

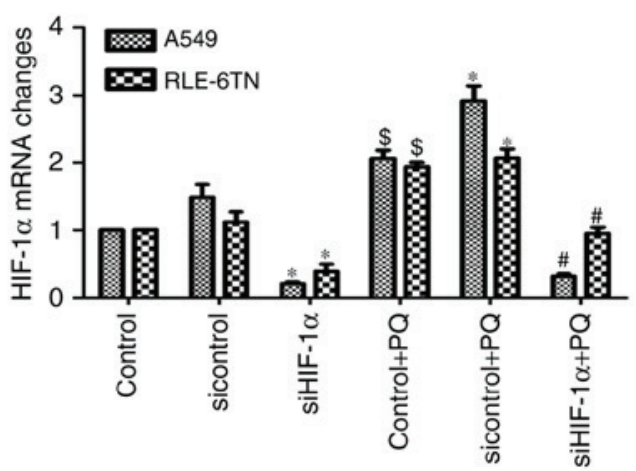

B

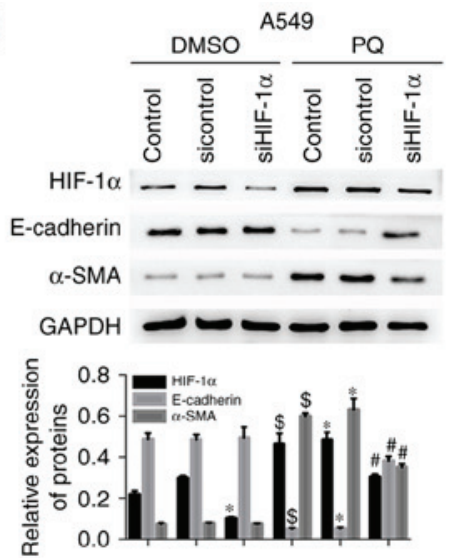

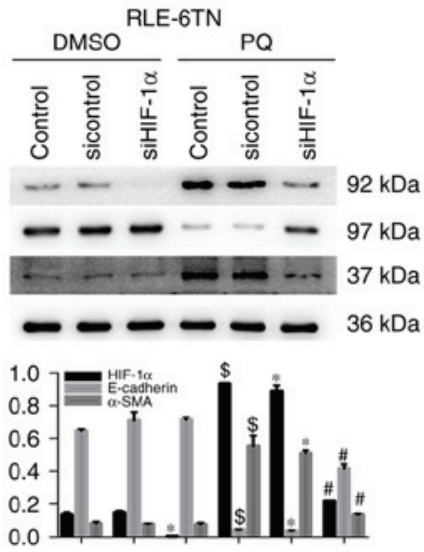
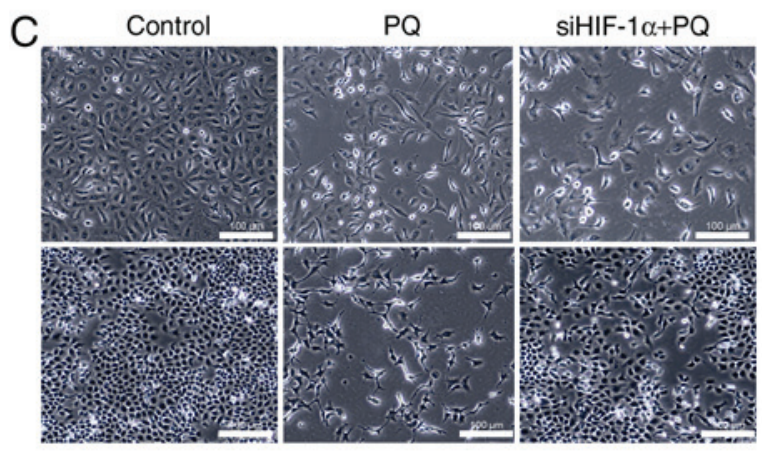

E
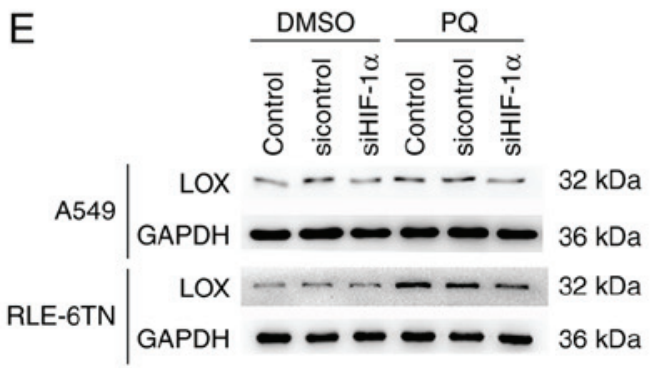
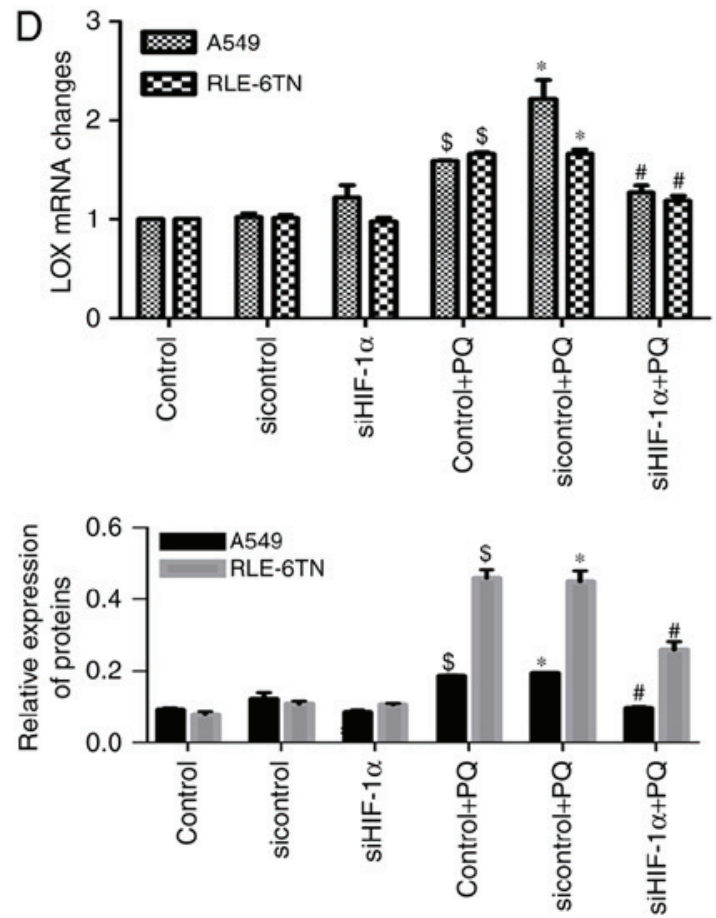

Figure 2. HIF-1 $\alpha$ ameliorated the degree of PQ-induced epithelial-to-mesenchymal transition and LOX expression. (A) HIF-1 $\alpha$ mRNA levels in HIF-1 $\alpha$-silenced cell lines was detected by RT-qPCR. (B) Protein levels of HIF-1 $\alpha$, E-cadherin, $\alpha$-SMA and GAPDH were detected by western blotting. GAPDH served as a loading control. (C) Morphological changes were detected using a phase-contrast microscope. Scale bars, $100 \mu \mathrm{m}$. (D) The level of LOX mRNA in both HIF-1 $\alpha$-silenced cell lines was detected using RT-qPCR. (E) LOX and GAPDH protein levels were detected by western blotting. ${ }^{\mathrm{S}} \mathrm{P}<0.05 \mathrm{vs}$. control; ${ }^{*} \mathrm{P}<0.05$ vs. sicontrol; ${ }^{~} \mathrm{P}<0.05$ vs. sicontrol + PQ. HIF-1 $\alpha$, hypoxia-inducible factor-1 $\alpha$; LOX, lysyl oxidase; PQ, paraquat; LOX, lysyl oxidase; RT-qPCR, reverse transcription-quantitative polymerase chain reaction; $\alpha$-SMA, $\alpha$-smooth muscle actin; DMSO, dimethyl sulfoxide.

\section{Discussion}

PQ accumulates in the lungs and eventually leads to PF; however, its molecular mechanisms are complex and remain unclear (2-4,7). EMT is known to have an important function in PF $(4,7,23)$. As demonstrated in recent studies by the present authors, EMT occurs in PQ-induced PF and may be modulated by HIF-1 $\alpha$ or LOX $(8,9)$. However, the interaction between HIF-1 $\alpha$ and LOX remains unclear. Therefore, the association between HIF-1 $\alpha$ and LOX was investigated, as was the pathway that regulates PQ-induced EMT. It was demonstrated that HIF-1 $\alpha$ may modulate PQ-induced EMT via the LOX/ $\beta$-catenin pathway.

LOX is a downstream target gene of HIF-1 $\alpha$, and a number of previous gene profiling studies have confirmed that LOX expression is upregulated by HIF-1 $\alpha$ (24-27). LOX is also an important regulator of hypoxia-induced tumor progression in a variety of cancers via a HIF-1 $\alpha$-dependent mechanism $(19,28)$. However, the correlation between HIF-1 $\alpha$ and LOX in fibrosis remains unexplored. In the present study, HIF-1 $\alpha$ and LOX expression were significantly increased in the model of PQ-induced PF. E-cadherin expression was decreased, and $\alpha$-SMA expression was significantly increased following PQ treatment, which confirmed that HIF-1 $\alpha$, LOX and EMT are associated with PQ-induced PF. Furthermore, HIF-1 $\alpha$ silencing downregulated the expression of LOX mRNA and protein. The expression of EMT markers was also reversed following HIF-1 $\alpha$ silencing, as $\alpha$-SMA expression decreased, and E-cadherin expression increased. In addition, changes in cellular morphology were alleviated following HIF-1 $\alpha$ silencing, which indicated that the degree of PQ-induced EMT was alleviated following HIF-1 $\alpha$ silencing. Therefore, HIF-1 $\alpha$ serves an important role 

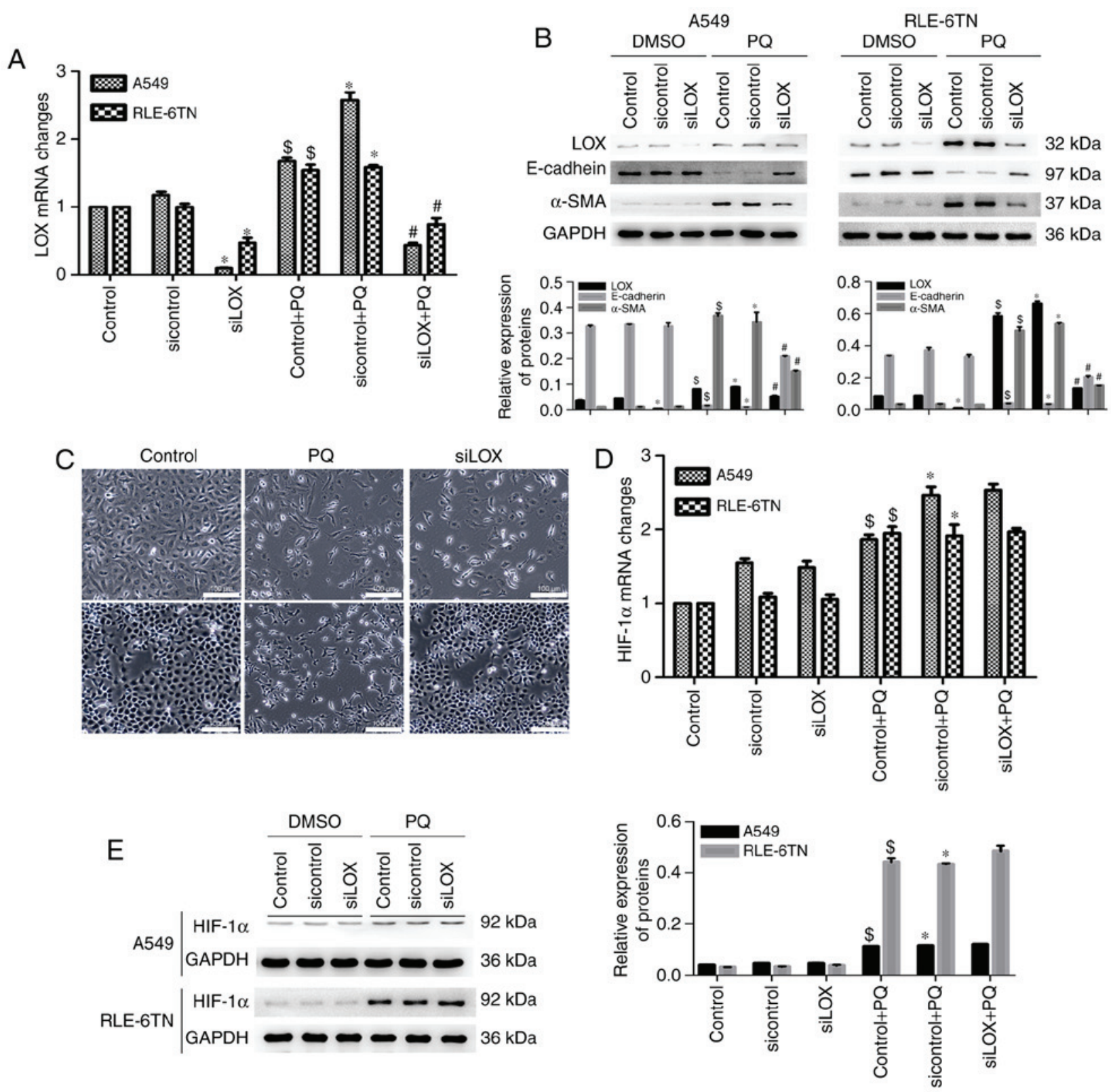

Figure 3. LOX reduced the degree of PQ-induced epithelial-to-mesenchymal transition and had no effect on HIF-1 $\alpha$ expression. (A) The level of LOX mRNA in both LOX-silenced cell lines was detected by RT-qPCR. (B) Levels of LOX, E-cadherin, $\alpha$-SMA and GAPDH proteins were detected by western blotting. GAPDH served as a loading control. (C) Morphological changes were detected using a phase-contrast microscope. Scale bars, $100 \mu \mathrm{m}$. (D) The level of HIF-1 $\alpha$ mRNA in both LOX-silenced cell lines was detected by RT-qPCR. (E) Levels of HIF-1 $\alpha$ and GAPDH proteins were detected by western blotting. ${ }^{\$} \mathrm{P}<0.05$ vs. control; "P<0.05 vs. sicontrol; " $\mathrm{P}<0.05$ vs. sicontrol + PQ. LOX, lysyl oxidase; PQ, paraquat; HIF-1 $1 \alpha$, hypoxia-inducible factor-1 $\alpha$; RT-qPCR, reverse transcription-quantitative polymerase chain reaction; $\alpha$-SMA, $\alpha$-smooth muscle actin; DMSO, dimethyl sulfoxide.

in modulating EMT by activating LOX in PQ-induced PF. This result is consistent with findings from previous studies, which demonstrated that HIF-1 $\alpha$ promotes EMT by upregulating LOX expression in ovarian and renal cancers $(27,29)$. It was also confirmed that LOX may be a target of HIF-1 $\alpha$ in PQ-induced $\mathrm{PF}$ and in tumors by modulating EMT.

In addition to acting as a HIF-1 $\alpha$-responsive gene, LOX may have more complex functions. According to a previous study by Pez et al (21), LOX and HIF-1 $\alpha$ act synergistically to promote colon cancer cell proliferation and tumor formation. As previously demonstrated by Ji et al (20), LOX silencing downregulates the protein expression of HIF- $1 \alpha$ in epithelial ovarian cancer cells. These findings indicated that LOX and HIF- $1 \alpha$ may bidirectionally regulate PQ-induced EMT.
However, in the present study, LOX silencing did not induce changes in the protein and mRNA levels of HIF-1 $\alpha$. However, the expression of EMT markers was ameliorated following LOX silencing. In addition, changes in cellular morphology were alleviated following LOX silencing. Therefore, the degree of PQ-induced EMT was alleviated following LOX silencing in vitro. This finding is consistent with other previously published results $(27,29)$ which reported that LOX inhibition did not prevent HIF-1 $\alpha$ upregulation. Furthermore, LOX is only an intermediate signaling molecule that mediates HIF-1 $\alpha$-promoted PQ-induced EMT.

$\beta$-catenin is a protein located in cytoplasmic plaques that serves a major role in EMT. $\beta$-catenin has been used as a marker of EMT in a number of studies of embryonic development, 
A
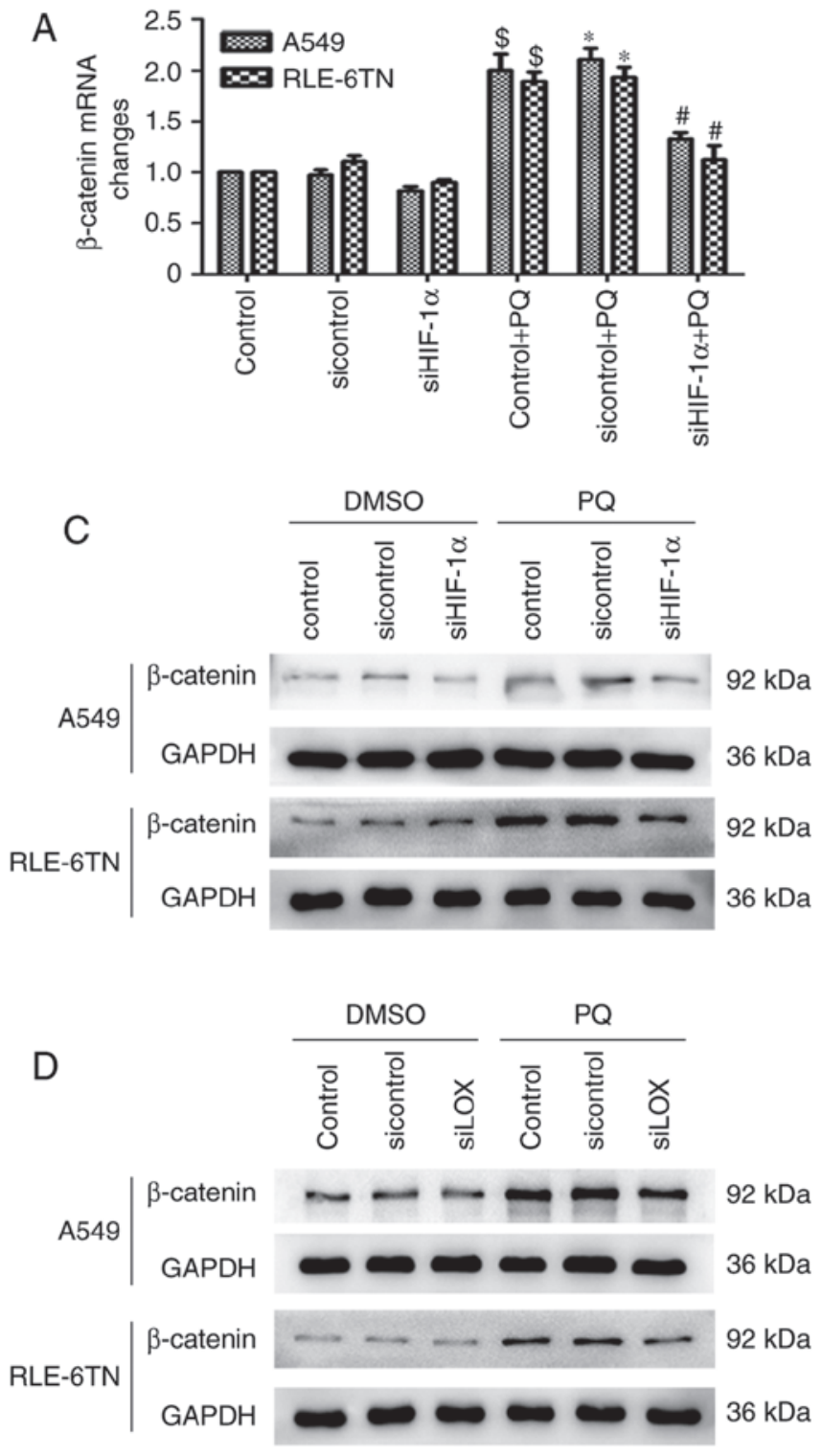

B
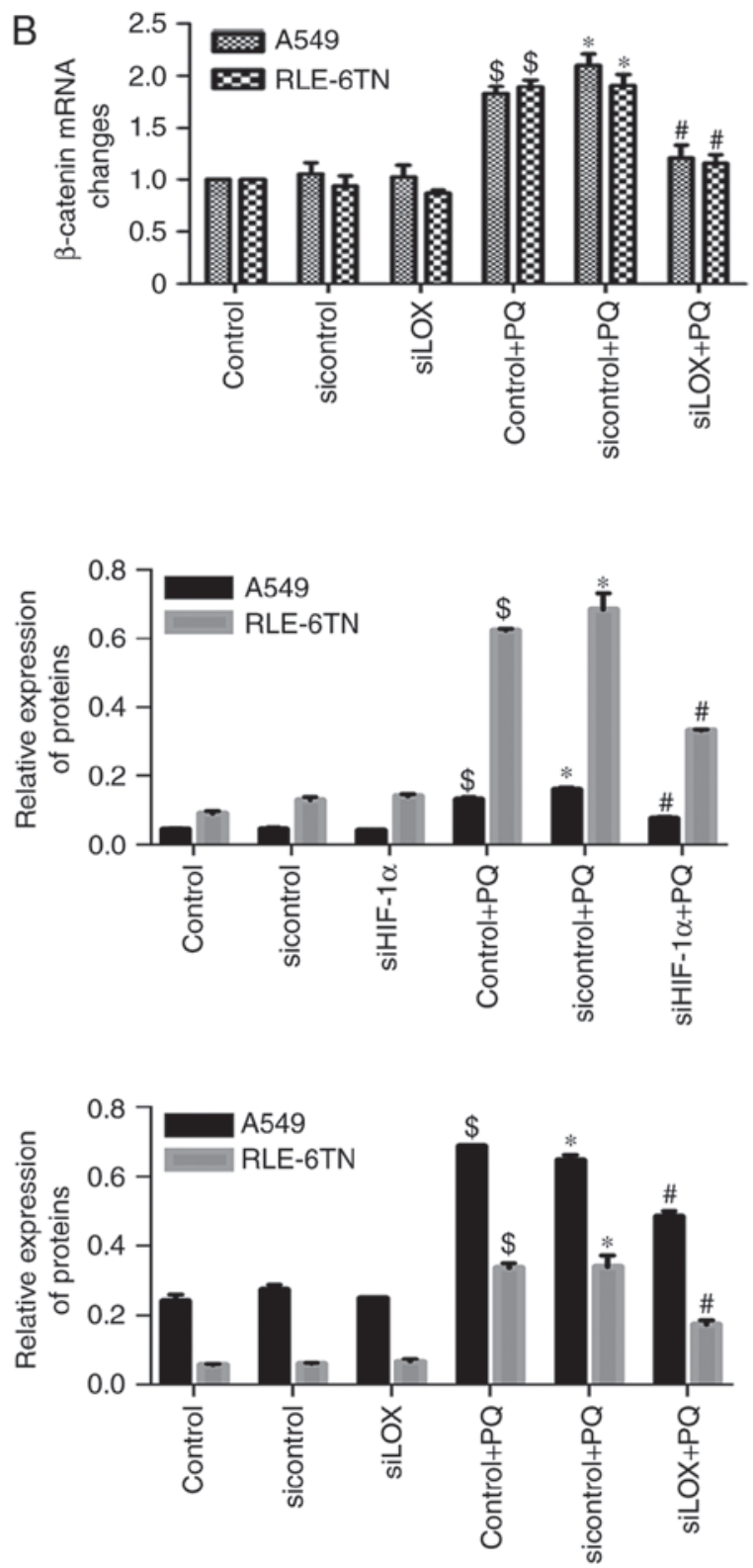

Figure 4. Inhibition of HIF-1 $\alpha$ and LOX decreases $\beta$-catenin expression in vitro. Levels of $\beta$-catenin mRNA in (A) HIF-1 $\alpha$ - and (B) LOX-silenced A549 and RLE-6TN cells were detected using reverse transcription-quantitative polymerase chain reaction. The expression of $\beta$-catenin protein in (C) HIF-1 $\alpha$ - and (D) LOX-silenced cells was detected using western blotting. GAPDH served as a loading control. ${ }^{\$} \mathrm{P}<0.05$ vs. control; ${ }^{*} \mathrm{P}<0.05$ vs. sicontrol; ${ }^{*} \mathrm{P}<0.05$ vs. sicontrol + PQ. HIF-1 $\alpha$, hypoxia-inducible factor-1 $\alpha$; LOX, lysyl oxidase; DMSO, dimethyl sulfoxide; PQ, paraquat.

cancer, and fibrosis (30-33). According to previous studies, $\beta$-catenin is associated with EMT during renal fibrosis (34) and fibrosis in other organs $(35,36)$. In addition, $\beta$-catenin participates in the development of PF by transforming A549 cells into fibroblasts $(23,37)$. As demonstrated previously, HIF- $1 \alpha$ is positively correlated with $\beta$-catenin in rat models, and HIF- $1 \alpha$ regulates EMT through the $\beta$-catenin pathway $(9,38)$. $\beta$-catenin mRNA and protein levels were significantly decreased when HIF- $1 \alpha$ and LOX were silenced in the present study, which suggests that $\mathrm{HIF}-1 \alpha$ regulates $\mathrm{PQ}$-induced EMT through the $\mathrm{LOX} / \beta$-catenin pathway.

The present study aimed to research the role of EMT in the development of PQ-induced pulmonary fibrosis. A549 cells retain the feature of type II alveolar epithelial cells even though they are a type of cancer cell. RLE-6TN cells were type II rat alveolar epithelial cells. These two cell types are widely used to study the mechanism of pulmonary fibrosis, therefore they were each selected for use within the present study to give a more comprehensive investigation. In the present study it was confirmed that EMT served a role in PQ-induced pulmonary fibrosis and may be modulated by HIF-1 $\alpha$ or LOX. HIF-1 $\alpha$ may modulate PQ-induced EMT via the $\mathrm{LOX} / \beta$-catenin pathway.

In conclusion, HIF-1 $\alpha$ unidirectionally upregulates LOX expression in PQ-induced EMT. The mechanism may be associated with HIF- $1 \alpha$-induced LOX expression, which subsequently increases $\beta$-catenin levels, induces EMT and ultimately leads to the development of PQ-induced PF. Therefore, HIF-1 $\alpha$ may be a potential target for restraining the development and exacerbation of PF induced by PQ. 


\section{Acknowledgements}

The present study was supported by grants from the National Natural Science Foundation of China (grant nos. 81602873 and 81502829) and the Key and Weak Subject Construction Project of the Shanghai Health and Family Planning System (grant no. 2016ZB0205).

\section{References}

1. Gil HW,Hong JR, Jang SH and Hong SY: Diagnostic and therapeutic approach for acute paraquat intoxication. J Korean Med Sci 29: 1441-1449, 2014.

2. Xu L, Xu J and Wang Z: Molecular mechanisms of paraquat-induced acute lung injury: A current review. Drug Chem Toxicol 37: 130-134, 2014.

3. Dinis-Oliveira RJ, Duarte JA, Sanchez-Navarro A, Remiao F, Bastos ML and Carvalho F: Paraquat poisonings: Mechanisms of lung toxicity, clinical features, and treatment. Crit Rev Toxicol 38 13-71,2008.

4. Nieto MA, Huang RY, Jackson RA and Thiery JP: EMT: 2016. Cell 166: 21-45, 2016.

5. Stone RC, Pastar I, Ojeh N, Chen V, Liu S, Garzon KI and Tomic-Canic M: Epithelial-mesenchymal transition in tissue repair and fibrosis. Cell Tissue Res 365: 495-506, 2016.

6. Bartis D, Mise N, Mahida RY, Eickelberg O and Thickett DR: Epithelial-mesenchymal transition in lung development and disease: Does it exist and is it important? Thorax 69: 760-765, 2014.

7. Kage $\mathrm{H}$ and Borok Z: EMT and interstitial lung disease: A mysterious relationship. Curr Opin Pulm Med 18: 517-523, 2012.

8. Wang J, Zhu Y, Tan J, Meng X, Xie H and Wang R: Lysyl oxidase promotes epithelial-to-mesenchymal transition during paraquat-induced pulmonary fibrosis. Mol Biosyst 12: 499-507, 2016.

9. Zhu Y, Tan J, Xie H, Wang J, Meng X and Wang R: HIF-1alpha regulates EMT via the Snail and beta-catenin pathways in paraquat poisoning-induced early pulmonary fibrosis. J Cell Mol Med 20 688-697, 2016.

10. Masoud GN and Li W: HIF-1alpha pathway: Role, regulation and intervention for cancer therapy. Acta Pharm Sin B 5: 378-389, 2015.

11. Balamurugan K: HIF-1 at the crossroads of hypoxia, inflammation, and cancer. Int J Cancer 138: 1058-1066, 2016.

12. Zhou G, Dada LA, Wu M, Kelly A, Trejo H, Zhou Q, Varga J and Sznajder JI: Hypoxia-induced alveolar epithelial-mesenchymal transition requires mitochondrial ROS and hypoxia-inducible factor 1 . Am J Physiol Lung Cell Mol Physiol 297: L1120-1130, 2009.

13. Darby IA and Hewitson TD: Hypoxia in tissue repair and fibrosis. Cell Tissue Res 365: 553-562, 2016.

14. Wang RL, Tang X, Wu X, Xu R, Yu KL and Xu K: The relationship between HIF-1 $\alpha$ expression and the early lung fibrosis in rats with acute paraquat poisoning. Zhonghua Lao Dong Wei Sheng Zhi Ye Bing Za Zhi 30: 273-277, 2012 (In Chinese).

15. Cox TR, Bird D, Baker AM, Barker HE, Ho MW, Lang G and Erler JT: LOX-mediated collagen crosslinking is responsible for fibrosis-enhanced metastasis. Cancer Res 73: 1721-1732, 2013.

16. Lopez B, Gonzalez A, Hermida N, Valencia F, de Teresa E and Diez J: Role of lysyl oxidase in myocardial fibrosis: From basic science to clinical aspects. Am J Physiol Heart Circ Physiol 299: H1-9, 2010.

17. Deng S, Jin T, Zhang L, Bu H and Zhang P: Mechanism of tacrolimus-induced chronic renal fibrosis following transplantation is regulated by ox-LDL and its receptor, LOX-1. Mol Med Rep 14: 4124-4134, 2016.

18. Cheng T, Liu Q, Zhang R, Zhang Y, Chen J, Yu R and Ge G: Lysyl oxidase promotes bleomycin-induced lung fibrosis through modulating inflammation. J Mol Cell Biol 6: 506-515, 2014.

19. Erler JT, Bennewith KL, Nicolau M, Dornhöfer N, Kong C, Le QT, Chi JT, Jeffrey SS and Giaccia AJ: Lysyl oxidase is essential for hypoxia-induced metastasis. Nature 440: 1222-1226, 2006.
20. Ji F, Wang Y, Qiu L, Li S, Zhu J, Liang Z, Wan Y and Di W: Hypoxia inducible factor $1 \alpha$-mediated LOX expression correlates with migration and invasion in epithelial ovarian cancer. Int J Oncol 42: 1578-1588, 2013.

21. Pez F, Dayan F, Durivault J, Kaniewski B, Aimond G, Le Provost GS, Deux B, Clézardin P, Sommer P, Pouysségur J and Reynaud C: The HIF-1-inducible lysyl oxidase activates HIF-1 via the Akt pathway in a positive regulation loop and synergizes with HIF-1 in promoting tumor cell growth. Cancer Res 71: 1647-1657, 2011.

22. Livak KJ and Schmittgen TD: Analysis of relative gene expression data using real-tie quantitative PCR and the 2(-Delta Delta C(T)) Method. Methods 25: 402-408, 2001.

23. Lamouille S, Xu J and Derynck R: Molecular mechanisms of epithelial-mesenchymal transition. Nat Rev Mol Cell Biol 15: 178-196, 2014.

24. Elvidge GP, Glenny L, Appelhoff RJ, Ratcliffe PJ, Ragoussis J and Gleadle JM: Concordant regulation of gene expression by hypoxia and 2-oxoglutarate-dependent dioxygenase inhibition: The role of HIF-1alpha, HIF-2alpha, and other pathways. J Biol Chem 281: $15215-15226,2006$.

25. Goto TM, Arima Y, Nagano O and Saya H: Lysyl oxidase is induced by cell density-mediated cell cycle suppression via RB-E2F1-HIF-1 $\alpha$ axis. Cell Struct Funct 38: 9-14, 2013.

26. Yang X, Li S, Li W, Chen J, Xiao X, Wang Y, Yan G and Chen L: Inactivation of lysyl oxidase by $\beta$-aminopropionitrile inhibits hypoxia-induced invasion and migration of cervical cancer cells. Oncol Rep 29: 541-548, 2013.

27. Wang Y, Ma J, Shen H, Wang C, Sun Y, Howell SB and Lin X: Reactive oxygen species promote ovarian cancer progression via the

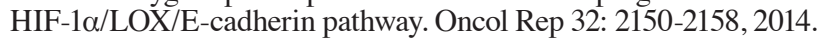

28. Reynaud C, Ferreras L, Di Mauro P, Kan C, Croset M, Bonnelye E, Pez F, Thomas C, Aimond G, Karnoub AE, et al: Lysyl oxidase is a strong determinant of tumor cell colonization in bone. Cancer Res 77: 268-278, 2017.

29. Schietke R, Warnecke C, Wacker I, Schödel J, Mole DR, Campean V, Amann K, Goppelt-Struebe M, Behrens J, Eckardt KU and Wiesener MS: The lysyl oxidases LOX and LOXL2 are necessary and sufficient to repress E-cadherin in hypoxia: Insights into cellular transformation processes mediated by HIF-1. J Biol Chem 285: 6658-6669, 2010.

30. Zeisberg M and Neilson EG: Biomarkers for epithelial-mesenchymal transitions. J Clin Invest 119: 1429-1437, 2009.

31. Xu L, Cui WH, Zhou WC, Li DL, Li LC, Zhao P, Mo XT, Zhang Z and Gao J: Activation of Wnt/B-catenin signalling is required for TGF- $\beta /$ Smad2/3 signalling during myofibroblast proliferation. J Cell Mol Med 21: 1545-1554, 2017.

32. Ji S, Deng H, Jin W, Yan P, Wang R, Pang L, Zhou J,Zhang J, Chen X, Zhao $X$ and Shen J: Beta-catenin participates in dialysate-induced peritoneal fibrosis via enhanced peritoneal cell epithelial-to-mesenchymal transition. FEBS Open Bio 7: 265-273, 2017.

33. Wang X, Dai W, Wang Y, Gu Q, Yang D and Zhang M: Blocking the Wnt/ $\beta$-catenin pathway by lentivirus-mediated short hairpin RNA targeting $\beta$-catenin gene suppresses silica-induced lung fibrosis in mice. Int J Environ Res Public Health 12: 10739-10754, 2015.

34. Martinez-Martinez E, Ibarrola J, Calvier L, Fernandez-Celis A, Leroy C, Cachofeiro V, Rossignol P and Lopez-Andres N: Galectin-3 blockade reduces renal fibrosis in two normotensive experimental models of renal damage. PLoS One 11: e0166272, 2016.

35. Hu BL, Shi C, Lei RE, Lu DH, Luo W, Qin SY, Zhou Y and Jianga HX: Interleukin-22 ameliorates liver fibrosis through miR-200a/beta-catenin. Sci Rep 6: 36436, 2016.

36. Lin JC, Kuo WW, Baskaran R, Chen MC, Ho TJ, Chen RJ, Chen YF, Vijaya Padma V, Lay IS and Huang CY: Enhancement of beta-catenin in cardiomyocytes suppresses survival protein expression but promotes apoptosis and fibrosis. Cardiol J 24: 195-205, 2017.

37. Anastas JN and Moon RT: WNT signalling pathways as therapeutic targets in cancer. Nat Rev Cancer 13: 11-26, 2013.

38. Xie H, Tan JT, Wang RL, Meng XX, Tang X and Gao S: Expression and significance of HIF-1alpha in pulmonary fibrosis induced by paraquat. Exp Biol Med (Maywood) 238: 1062-1068, 2013.

This work is licensed under a Creative Commons

Attribution-NonCommercial-NoDerivatives 4.0 International (CC BY-NC-ND 4.0) License. 